\title{
Selective Peak Enhancement and Suppression in Conductometric Ion Chromatography and Its Application to the Determination of Trace Levels of Orthophosphate in Environmental Samples
}

\author{
Kazuichi Hayakawa* ${ }^{* \dagger}$, Akio Kato*, Atsushi Yamamoto** and Motoichi Miyazaki* \\ * Faculty of Pharmaceutical Sciences, Kanazawa University, Takara-machi, Kanazawa 920, Japan \\ ** Toyama Institute of Health, Nakataikoyama, Kosugi-machi, Toyama 939-03, Japan
}

\begin{abstract}
In non-suppressed conductometric ion chromatography, when a carboxylic acid eluent of low $\mathrm{pH}$ is used, the signal intensity $\left(\Delta H_{\mathrm{C}}\right)$ is proportional to the sum of the conductivity change caused by changes in the sample concentration $\left(\Delta C_{s}\right)$ as well as conductivity changes caused by changes in the eluent concentration $\left(\Delta C_{\mathrm{e}}\right)$. This can be expressed as $\Delta H_{\mathrm{C}}=B\left(\Delta C_{\mathrm{s}}\right)+B\left(\Delta C_{\mathrm{e}}\right)$, where $B$ is a constant. In this equation, $\Delta C_{\mathrm{s}}$ is constant for any sample species. However, $\Delta C_{\mathrm{e}}$ varies with changes in the sample capacity factors. This value is positive when the sample elutes before the system peak $\left(k_{\mathrm{s}}^{\prime}<k_{\mathrm{e}}^{\prime}\right)$, and is negative when it elutes after the system peak $\left(k_{\mathrm{s}}^{>}>k_{\mathrm{e}}^{\prime}\right)$. Therefore, the sample peak intensity $\left(\Delta H_{\mathrm{C}}\right)$ is enhanced prior to the system peak and is suppressed after it. These effects increase the closer the sample and the system peaks. The effect of this theory was proved by a determination of orthophosphate using a TSK gel IC-Anion-PW column and a $0.75 \mathrm{mM}$ phthalic acid eluent. The detection limit of this method for orthophosphate $\left(1 \times 10^{-11} \mathrm{~mol}\right.$, with an $S / N$ of three) was smaller (by a factor of $10-30$ ) than those by previous conventional ion chromatographic methods. Also, the interference of chloride or nitrate, often observed in the previous methods, was removed by suppressing their peak intensities. When this method was applied for the determination of orthophosphate in environmental samples, other interfering compounds were effectively removed by a column-switching system attached to the ion chromatograph, and a trace level of the orthophosphate was determined.
\end{abstract}

Keywords Orthophosphate, conductometric ion chromatography, selective peak enhancement, selective peak suppression, column-switching, environmental sample

Ion chromatography (IC) ${ }^{1}$ is used as a standard determination method for ionic compounds in many fields. Recently, the popularity of non-suppressed IC has increased due to its easier handling and lower price. ${ }^{2}$ In non-suppressed IC, both conductometric and photometric detectors are used, since their different mechanisms and advantages complement each other. ${ }^{3,4}$ However, some problems still remain, one of which is a lack of sensitivity for several species of ions. Another is interfering peaks due to large amounts of coexisting compounds and the system peak.

We have previously reported theoretical considerations using photometric detection (PD) in nonsuppressed anion chromatography, with a weak acid eluent of low $\mathrm{pH}$. According to that theory, the sample peak is positive (i.e. absorbance increases) before the system peak, and is negative (absorbance decreases) after it. Their intensities increase the closer is the proximity to the system peak. Both dissociated and undissociated species of the weak acid eluent contribute to peak

$\dagger$ To whom correspondence should be addressed. formation. ${ }^{5}$ This theory was successfully applied in a simultaneous determination of acetic acid and several other trace organic acids in vinegars. ${ }^{6}$ Under the same conditions we found that the peak formation mechanism of conductometric detection (CD) differed from that of PD described above. The sample peak (i.e. a positive peak) is enhanced when eluting is carried out before the system peak, and is suppressed when eluting is carried out after it. This mechanism not only enhances the sample peak, but also suppresses the interfering peak.

Orthophosphate in environmental samples is closely related to eutrophication. Although several highperformance liquid-chromatographic and IC methods have been reported regarding the determination of orthophosphate $^{7-9}$, they are not sufficiently sensitive for environmental samples. Moreover, environmental samples often contain large amounts of coexisting compounds which interfere with the determination. These problems were completely resolved by the above theory with column-switching IC. ${ }^{10}$

The purpose of this report is to describe this selective peak-enhancement and suppression theory, as well as its 
effective use in the determination of orthophosphate in environmental samples.

\section{Theory}

According to our previous report ${ }^{5}$, the elution of sample anions from an anion exchange column causes a change in the eluent concentration. This change is expressed as the change in the eluent concentration $[\Delta \mathrm{E}]$ devided by the change in the concentration of sample species $[\Delta \mathrm{S}]$, over the area in which the peak elutes:

$$
[\Delta \mathrm{E}] /[\Delta \mathrm{S}]=k_{\mathrm{s}}^{\prime} /\left(k_{\mathrm{e}}^{\prime}-k_{\mathrm{s}}^{\prime}\right),
$$

where $k_{\mathrm{s}}^{\prime}$ and $k_{\mathrm{e}}^{\prime}$ are capacity factors of the sample and the system peaks, respectively. Equation (1) suggests that the eluent concentration increases when the sample elutes before the system peak $\left(k_{\mathrm{e}}^{\prime}>k_{\mathrm{s}}^{\prime}\right)$, and decreases when it elutes after the system peak $\left(k_{\mathrm{e}}^{\prime}<k_{\mathrm{s}}^{\prime}\right)$. The magnitude of this change increases the closer the proximity of these two peaks.

In $\mathrm{CD}$, both sample and eluent ions respond to the detector. If we suppose that the sample compound completely dissociates to monovalent anions and hydrogen ions, the signal intensity $\left(\Delta H_{\mathrm{C}}\right)$ can be expressed as follows:

$$
\Delta H_{\mathrm{C}}=B\left(\lambda_{\mathrm{s}}{ }^{-}+\lambda_{\mathrm{H}^{+}}\right)[\Delta \mathrm{S}]+B\left(\lambda_{\mathrm{e}^{-}}+\lambda_{\mathrm{H}^{+}}\right)[\Delta \mathrm{E}] I_{\mathrm{e}},
$$

where $I_{\mathrm{e}}$ is the dissociation degree of the eluent species; $\lambda_{\mathrm{e}}{ }^{-}, \lambda_{\mathrm{s}}{ }^{-}$and $\lambda_{\mathrm{H}^{+}}$are the equivalent conductivities of the eluent, sample and hydrogen ions, respectively; and $B$ is a constant. By substituting $[\Delta \mathrm{E}]$ with Eq. (1), $\Delta H_{\mathrm{C}}$ can be expressed as

$$
\begin{aligned}
\Delta H_{\mathrm{C}}= & B\left(\lambda_{\mathrm{s}}{ }^{-}+\lambda_{\mathrm{H}^{+}}\right)[\Delta \mathrm{S}] \\
& +B\left(\lambda_{\mathrm{e}}{ }^{-}+\lambda_{\mathrm{H}^{+}}\right) I_{\mathrm{e}}[\Delta \mathrm{S}] k_{\mathrm{s}}^{\prime} /\left(k_{\mathrm{e}}^{\prime}-k_{\mathrm{s}}^{\prime}\right) .
\end{aligned}
$$

Figure 1 shows a simplified model, assuming that samples I and II elute before the system peak and III and IV after the system peak. (a) shows the change in conductivity based only on the samples eluted, which is given by the first term in Eq. (3). (b) shows the profile based only on the change in the eluent concentration, which is given by the second term in Eq. (3). (c) shows the actual detector response, which is given by a sum of (a) and (b) (signals I', II', III' and IV' correspond to samples I, II, III and IV, respectively). Signals I' and II', which elute before the system peak, are enhanced by an increase in the eluent concentration. Likewise, signals III' and IV', which elute after the system peak, are suppressed by a decrease in the eluent concentration. The magnitude of these effects increases the closer the sample peaks to the system peak.

On the other hand, in PD using UV-absorbing eluents, elution of a non-UV-absorbing sample is detected by changes in the eluent concentration $([\Delta E])$, only. Since (a)

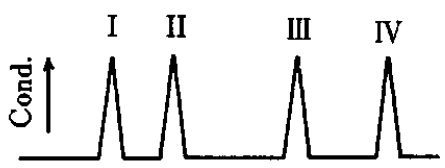

(b)

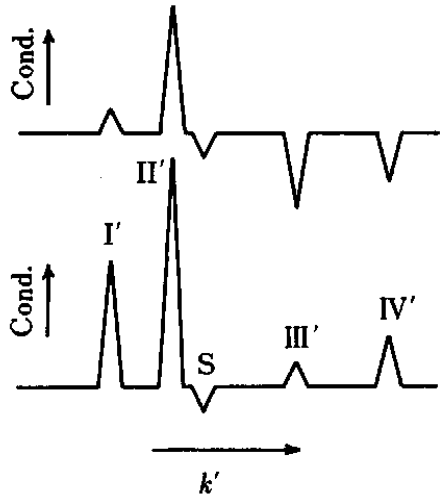

Fig. 1 Schematic profile models of non-suppressed conductometric ion chromatography. (a), conductivity change due to the elution of sample species (I, II, III and IV); (b), conductivity change due to the elution of the eluent; (c), actual chromatogram. Peaks $I^{\prime}-I^{\prime}$ ' correspond, respectively, to $\mathrm{I}-\mathrm{IV}$, and $\mathrm{S}$ to the system peak.

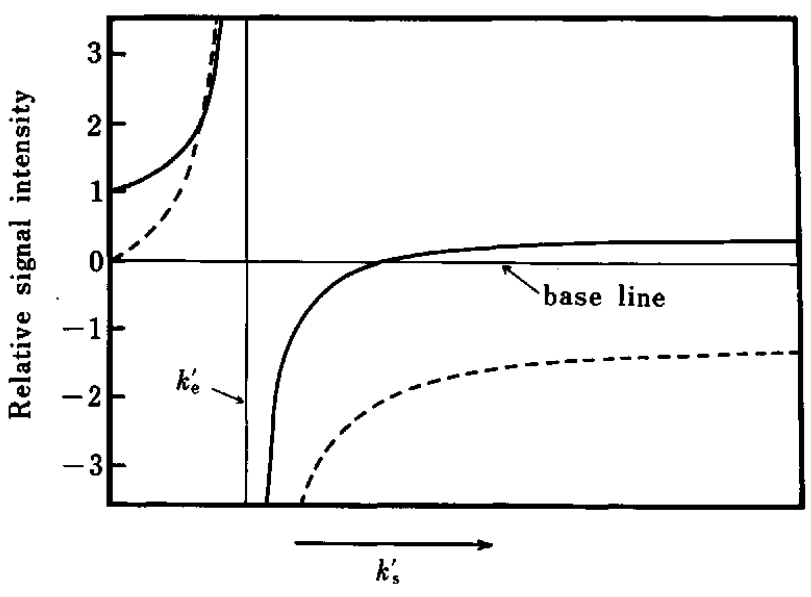

Fig. 2 Relative signal intensities of conductometric (-) and photometric (---) detection $v s$. capacity factor.

there is not any direct contribution from the sample ${ }^{3,12}$, the signal intensity $\left(\Delta H_{\mathrm{P}}\right)$ of the sample can be expressed as

$$
\Delta H_{\mathrm{P}}=A[\Delta \mathrm{S}] \lambda_{\mathrm{e}} k_{\mathrm{s}}^{\prime} /\left(k_{\mathrm{e}}^{\prime}-k_{\mathrm{s}}^{\prime}\right),
$$

where $\lambda_{e}$ is the molar absorptivity of the eluent species and $A$ is a constant. Figure 2 shows the signal intensitycapacity factor $\left(k^{\prime}\right)$ profiles for CD (solid line) and PD calculated from Eqs. (3) and (4), respectively. The former was calculated with $\lambda_{\mathrm{s}}{ }^{-}=\lambda_{\mathrm{e}}{ }^{-}$and $I_{\mathrm{e}}=0.5$. A positive sample peak is enhanced by both detection methods when $k_{s}^{\prime}$ is smaller than $k_{e}^{\prime}$, although the degree 
of enhancement varies between the two. However, a significant difference is observed when $k_{\mathrm{e}}^{\prime}<k_{\mathrm{s}}^{\prime}$. In PD, a negative peak formed by a decrease in the eluent concentration (eluent ravine), is always observed. Its magnitude increases as $\left(\boldsymbol{k}_{\mathrm{e}}^{\prime}-\boldsymbol{k}_{\mathrm{s}}^{\prime}\right)$ approaches zero. In $\mathrm{CD}$, on the other hand, the magnitude of the sample peak (i.e. a positive peak) decreases as the eluent ravine becomes deep. The peak is negative upon closing with the system peak. Figure 2 suggests that this selective peak enhancement and suppression achieved with CD are useful for the determination of anions at the trace level in the presence of large amounts of interfering ions. Although frequently in IC the elution order of ions can not always be changed, this technique may prove to be useful for several practical samples.

\section{Experimental}

\section{Chemicals and solutions}

All the chemicals used were of guaranteed commercial grade. Water was purified by a Millipore (Milford, USA) Milli-Q water purification system. The eluent was prepared by dissolving phthalic acid in water. The concentration used for the determination of orthophosphate was $0.75 \mathrm{mM}$. The carrier solution used in environmental sample analysis was water. Standard anion solutions were prepared by dissolving sodium salts of orthophosphate, chloride and nitrate in water. These solutions were all filtered through a membrane $(0.4 \mu \mathrm{m})$ before use.

\section{Environmental samples}

Sewage and seawater samples were collected in Shiomachi, Ishikawa, Japan (August 17, 1990) and filtered through a membrane $(0.4 \mu \mathrm{m})$.

\section{IC system and its conditions}

A schematic diagram of the full IC system used is

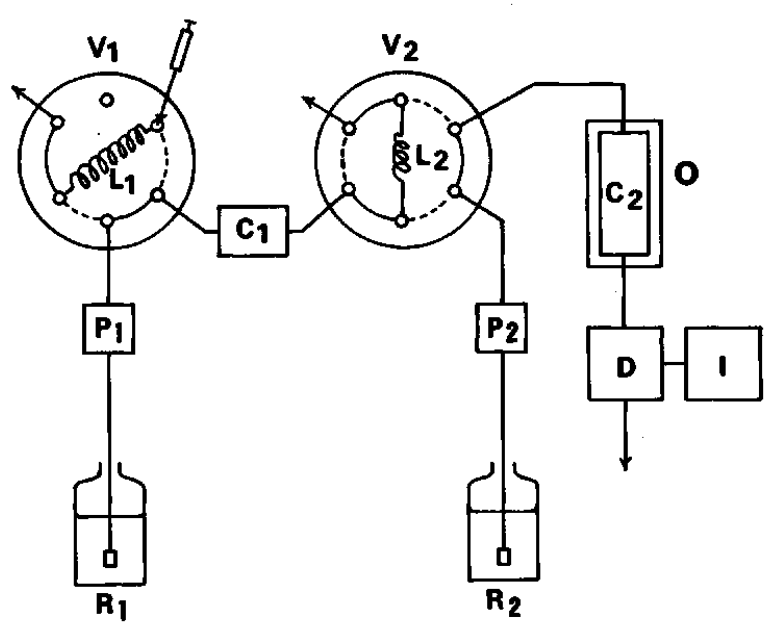

Fig. 3 Schematic diagram of IC with an on-line clean-up system. shown in Fig. 3. The IC system used in the fundamental study was a Shimadzu (Kyoto, Japan) HIC-6A nonsuppressed conductivity detection system comprising a Shimadzu LP-6A pump $\left(\mathbf{P}_{2}\right)$, a Rheodyne 7125 injector $\left(V_{2}\right)$ with a $20 \mu$ loop $\left(L_{2}\right)$, a Shimadzu CTO-6AS column oven (O), a Shimadzu CDD-6A conductivity detector (D) and a Shimadzu C-R3A integrator (I). The analytical column $\left(\mathrm{C}_{2}\right)$ was a Tosoh (Tokyo, Japan) TSK gel IC-Anion-PW (4.6 mm i.d. $\times 50 \mathrm{~mm}$, methacrylatebased, anion exchange capacity of $30 \pm 3 \mu \mathrm{eq} / \mathrm{ml}$ ) kept at $40^{\circ} \mathrm{C}$. The eluent in a reservoir $\left(R_{2}\right)$ was pumped into $\mathrm{C}_{2}$ at a flow rate of $1.2 \mathrm{ml} / \mathrm{min}$.

When environmental samples were used, an on-line clean-up system was attached to the above-mentioned IC system according to our previous results. ${ }^{11}$ The cleanup system comprised of a Kyowa (Tokyo, Japan) KHP010 pump $\left(P_{1}\right)$, a Rheodyne 7125 injector $\left(V_{1}\right)$ with a $300 \mu$ loop $\left(\mathrm{L}_{1}\right)$ and a remover column $\left(\mathrm{C}_{1}\right)$, where $\mathrm{L}_{2}$ is a heart-cutting loop of the treated solution. $C_{1}$ is a Tosoh TSK guard column IC-A (4.6 mm i.d. $\times 50 \mathrm{~mm})$. Water in a reservoir $\left(R_{1}\right)$ was pumped as a sample carrier at a flow rate of $1: 0 \mathrm{ml} / \mathrm{min}$.

The operation of the full IC system was as follows. When both $V_{1}$ and $V_{2}$ were at their initial positions (solid line in Fig. 3), both $\mathrm{C}_{1}$ and $\mathrm{C}_{2}$ were conditioned with the water and eluent, respectively. The sample solution was injected into $L_{1}$, and immediately sent to $C_{1}$ by switching $\mathrm{V}_{1}$ (dotted line). At $35 \mathrm{~s}$ after the switching, $\mathrm{V}_{2}$ was switched (dotted line). The eluate from $\mathrm{C}_{2}$ was detected with D. At $30 \mathrm{~min}$ after sample injection, both valves were returned to their initial positions.

\section{Results and Discussion}

Carboxylate solutions, such as phthalate, have been used as eluents in previous non-suppressed IC with CD. The $\mathrm{pH}$ of those eluents was often adjusted to 4 or more. Under these conditions, the increase in the eluent concentration was not observed over the area of sample elution, even when $k_{\mathrm{e}}^{\prime}<k_{\mathrm{s}}^{\prime}$. Moreover, the limiting ionic equivalent conductivity of orthophosphate $\left(36.0 \mathrm{~S} \mathrm{~cm}^{2}\right)$ was smaller than those of chloride $\left(76.4 \mathrm{~S} \mathrm{~cm}^{2}\right)$ and nitrate $\left(71.5 \mathrm{~S} \mathrm{~cm}^{2}\right) .^{13}$ This resulted in orthophosphate having the smallest peak intensity of the three anions.

According to the proposed theory, the orthophosphate peak is selectively enhanced when eluted just before the system peak, while both the chloride and nitrate peaks are suppressed when eluted after the system peak. Thus, their elution behaviors was examined on a TSK gel IC-Anion-PW column. Among the eluents tested, an aqueous solution of phthalic acid $\left(\mathrm{p} K_{\mathrm{a} 1}=2.76, \mathrm{p} K_{\mathrm{a} 2}\right.$ $=4.92$ ) was found to satisfy the above conditions. Figure 4 shows the capacity factors of the three anions and the system peak as a factor of phthalic acid concentration. In the range 0.5 to $1.3 \mathrm{mM}$ (the $\mathrm{pH}$ being 3.0 to 3.4 ), the system peak appears between orthophosphate and the other two anions. Although, according to the theory, the orthophosphate peak 


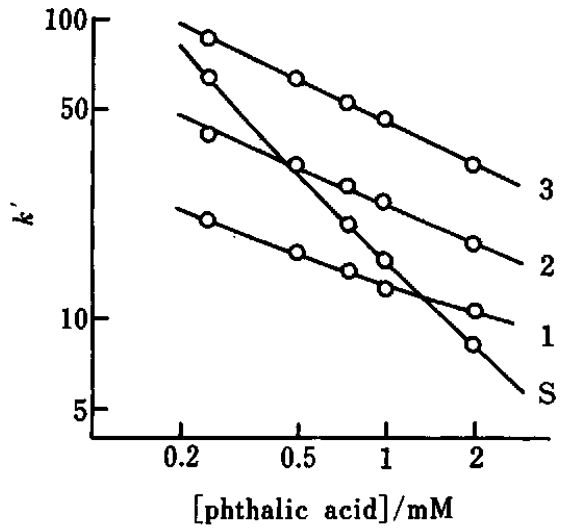

Fig. 4 Elution behaviors of the three anions and the system peak as a factor of the phthalic acid concentration on a TSK gel IC-Anion-PW column. S, system peak; 1, orthophosphate; 2 , chloride; 3 , nitrate.

Table 1 Detection limit of orthophosphate by several nonsuppressed ion chromatographic methods

\begin{tabular}{cc}
\multicolumn{1}{c}{$\begin{array}{c}\text { Detection method } \\
\text { (Conditions) }\end{array}$} & Detection limit ${ }^{\mathrm{a}}$ \\
\hline $\begin{array}{l}\text { Previous methods } \\
\text { Conductometric } \\
\text { Eluent, } 2.0 \mathrm{mM} \text { tartaric acid; } \\
\text { column, TSK gel IC-Anion-SW }\end{array}$ & $1 \times 10^{-10} \mathrm{~mol}$ \\
Indirect photometric \\
$\begin{array}{l}\text { Eluent, 0.5 mM sodium hydrogen- } \\
\text { phthalate-0.5 mM HEPES (pH 6.6); } \\
\text { column, TSK gel IC-Anion-PW }\end{array}$ \\
$\begin{array}{l}\text { Proposed method (conductometric) } \\
\text { Eluent, 0.75 mM phthalic acid; } \\
\text { column, TSK gel IC-Anion-PW }\end{array}$ \\
\hline
\end{tabular}

a. $S / N=3$. A Shimadzu HIC-6A system equipped with a Shimadzu SPD 6AV (UV detector) was used in this comparison.

becomes larger the closer to the system peak, the resolution $(R)$ of the two peaks becomes smaller. In the following experiments, $0.75 \mathrm{mM}$ phthalic acid (with $R>2$ ) was used as the eluent.

The detection limit $(S / N=3)$ of orthophosphate by the proposed method was $1 \times 10^{-11} \mathrm{~mol}$. The sensitivity of this method is $10-30$ times as high as those of previous non-suppressed IC methods (Table 1). Figure 5 shows a standard chromatogram of equal amounts of orthophosphate, chloride and nitrate. After subsequent enhancement and suppression, the orthophosphate peak becomes about 13 and 9 times as high as that of the chloride and nitrate, respectively.

By using an IC system without a column-switching clean-up system, orthophosphate in such samples as seawater and river water were determined. However, such samples as sewage showed a base line drift which interfered with the determination of orthophosphate.

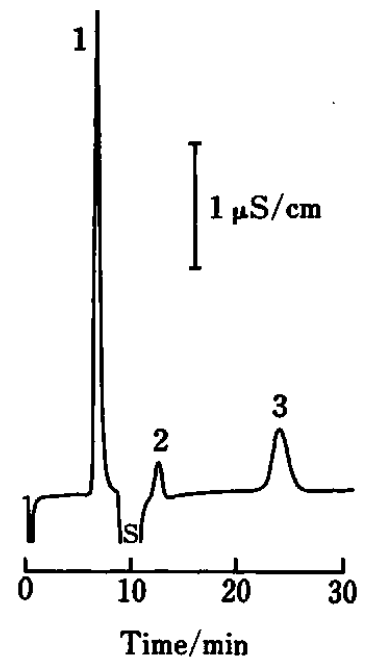

Fig. 5 Standard chromatogram of the three anions (5.0X $10^{-9}$ mol each). Peaks: 1 , orthophosphate; 2, chloride; 3 , nitrate; $S$, system peak.

Although the compounds which caused this problem were still unknown, we found that they were retained on a TSK guard column IC-A, and eluted after analyte anions when water was used as a carrier. However, peak-broadening occurred when this column was inserted just before the analytical column. We reported that the heart-cutting technique reduced the peakbroadening completely after an on-line removal column treatment. ${ }^{11}$ Therefore, a switching valve $\left(V_{2}\right)$ equipped with a cutting loop was used between $C_{1}$ and $C_{2}$ in the full system, as shown in Fig. 3. The switching time of $V_{2}$ was set at $35 \mathrm{~s}$ to give the highest orthophosphate peak. Other operating conditions of this system were optimized according to our report. ${ }^{11}$

Utilizing this full IC system, orthophosphate at $\mu \mathrm{M}$ and sub $\mu \mathrm{M}$ levels in sewage, seawater and river water
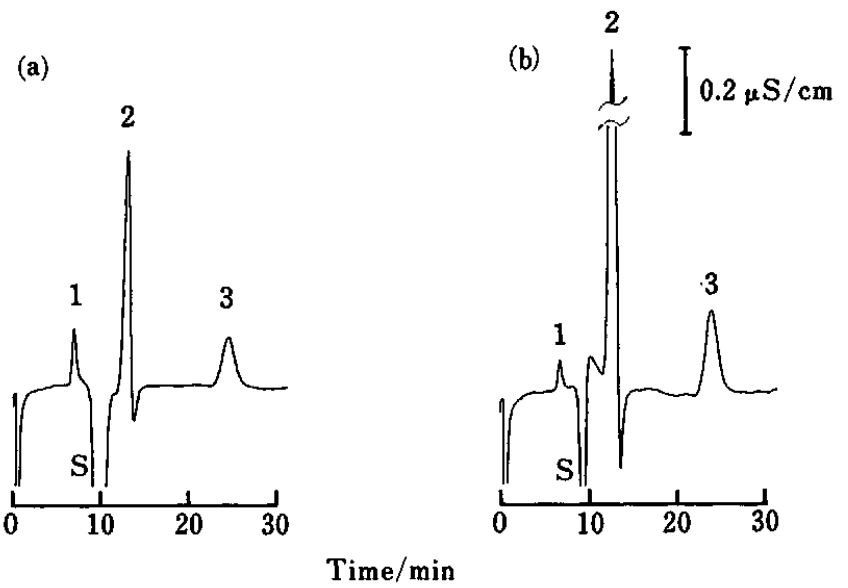

Fig. 6 Chromatograms of (a) sewage and (b) seawater. The peak numbers are the same as in Fig. 4. 
samples were determined without any interference. Typical chromatograms of sewage and seawater samples are shown in Fig. 6. There are no peaks which interfere with the determination. The orthophosphate concentrations in the two samples were 12.9 and $8.3 \mu \mathrm{M}$, respectively. These levels were almost as low as, or lower than, the detection limits of the previous IC methods listed in Table 1.

This work was partly supported by a Grant-in-Aid for General Scientific Research No. 02670975 from the Ministry of Education; Science and Culture, Japan.

\section{References}

1. H. Small, T. S. Stevens and W. C. Bauman, Anal. Chem., 47, 1801 (1975).

2. D. T. Gjerde, J. S. Fritz and G. Schmuckler, J. Chromatogr., 187, 35 (1979).

3. H. Small and T. E. Miller, Jr., Anal. Chem., 54, 462 (1982).

4. S. Nakamura, N. Imaizumi, K. Hayakawa and $M$.
Miyazaki, Bunseki Kagaku, 38, 573 (1989).

5. A. Yamamoto, A. Matsunaga, M. Ohto, E. Mizukami, K. Hayakawa and M. Miyazaki, J. Chromatogr., 481, 323 (1989).

6. A. Yamamoto, A. Matsunaga, E. Mizukami, K. Hayakawa and M. Miyazaki, Eisei Kagaku, 36, 332 (1990).

7. D. P. Lee, J. Chromatogr. Sci., 22, 327 (1984).

8. Y. Baba, N. Yoza and S. Ohashi, J. Chromatogr., 348, 27 (1985).

9. M. C. Mehra and C. Pelletier, Anal. Sci., 6, 431 (1990).

10. I. Yoshida, K. Hayakawa and M. Miyazaki, Nippon Kagaku Kaishi, 1986, 1046.

11. K. Hayakawa, K. Nomura and M. Miyazaki, Anal. Sci., 7, 967 (1991).

12. M. Miyazaki and K. Hayakawa, "Atarashii Ion Chromatography no Tehodoki (Introduction to New Ion Chromatography, in Japanese)", Nankodo, Tokyo, 1986.

13. R. A. Robinson and R. H. Stokes, "Electrolyte Solution", pp. 463-465, Butterworths, London, 1959.

(Received September 6, 1991) (Accepted November 12, 1991) 